\title{
An interior penalty method for a finite-dimensional linear complementarity problem in financial engineering
}

\author{
Song Wang · Kai Zhang
}

Received: date / Accepted: date

\begin{abstract}
In this work we study an interior penalty method for a finitedimensional large-scale Linear Complementarity Problem (LCP) arising often from the discretization of stochastic optimal problems in financial engineering. In this approach, we approximate the LCP by a nonlinear algebraic equation containing a penalty term linked to the logarithmic barrier function for constrained optimization problems. We show that the penalty equation has a solution and establish a convergence theory for the approximate solutions. A smooth Newton method is proposed for solving the penalty equation and properties of the Jacobian matrix in the Newton method have been investigated. Numerical experimental results using two non-trivial test examples are presented to demonstrate the rates of convergence, efficiency and usefulness of the method for solving practical problems.
\end{abstract}

Keywords Variational inequality · stochastic optimal control · American option pricing · HJB equation · linear complementarity problem · interior penalty method.

\section{Introduction}

Many real-world phenomena in engineering, physics, mechanics, economics and financial engineering can be formulated as differential Linear Complementarity Problems (LCPs) of the form

$$
L_{1} u \leq f, \quad L_{2} u \leq u^{*} \quad \text { and } \quad\left(L_{1} u-f\right) \cdot\left(L_{2} u-u^{*}\right)=0
$$

Song Wang

Department of Mathematics \& Statistics, Curtin University, GPO Box U1987, Perth WA6845, Australia. E-mail: song.wang@curtin.edu.au

Kai Zhang

Business School, Shenzhen University, Shenzhen, China, 518060 E-mail: kaizhang@szu.edu.cn 
defined on a given solution domain in space and time with appropriate boundary and initial/terminal conditions which are also bounded above by $u^{*}$, where $L_{1}$ and $L_{2}$ are linear differential operators and $f$ and $u^{*}$ are known functions $[2,18,13,11]$. This problem may also be viewed as a Hamilton-Jacobi-Bellman equation which often arises from stochastic optimal control problems in financial engineering, in particular, financial derivative valuation $[35,6,8,33,38$, 3]. A typical example is the American option valuation problem in which the value $V$ of an American option on an asset with its price following a geometric Brownian motion is governed by the following infinite-dimensional LCP

$$
\begin{aligned}
L V:=-\frac{\partial V}{\partial t}-\frac{1}{2} \sigma^{2} S^{2} \frac{\partial^{2} V}{\partial S^{2}}-r S \frac{\partial V}{\partial S}+r V & \geq 0, \\
V(S, t)-V^{*}(S) & \geq 0, \\
L V(S, t) \cdot\left(V(S, t)-V^{*}(S)\right) & =0
\end{aligned}
$$

for $(S, t) \in\left(0, S_{\max }\right) \times[0, T)$ with a given terminal/payoff condition $V^{*}(t)$ and appropriate boundary conditions, where $\sigma$ denotes the volatility of the underlying asset and $r$ the risk-free interest rate. Other examples of LCPs arising in stochastic optimal control in financial engineering including the cases that $L_{2}$ is not an identity can be found in $[6,8,20,3]$.

Differential LCPs in infinite dimensions are normally not solvable analytically and numerical approximations to them are usually sought in practice. Various discretization methods have been developed for differential LCPs such as those in $[5,28,9,19,20,34,4]$, just to name a few. Applying a discretization scheme to such a problem yields a finite-dimensional LCP of the following form:

Problem 1 Find $x \in \mathbb{R}^{n}$ such that

$$
A x \leq b, \quad C x \leq d, \quad(A x-b)^{\top}(C x-d)=0,
$$

where $A$ and $C$ are $n \times n$ matrices, and $b$ and $c$ are known vectors in $\mathbb{R}^{n}$ with $n \gg 1$.

A square matrix is called an $M$-matrix if the real parts of all its eigenvalues are positive and all its off-diagonal entries are non-positive. If $Z$ is an $M$ matrix, then $Z$ is invertible and $Z^{-1} \geq 0$. A discretization scheme such as any of the ones mentioned above is designed so that the coefficient matrices of the resulting linear algebraic system are positive-definite $M$-matrices, but usually not symmetric. Therefore, we make the following assumptions on the system matrices $A$ and $C$ in Problem 1:

A1. $A$ is positive definite, i.e., there exists a constant $a_{0}>0$ such that $y^{\top} A y \geq$ $a_{0}\|y\|^{2}, \quad \forall y \in \mathbb{R}^{n}$, where $\|\cdot\|$ denotes the Euclidean norm on $\mathbb{R}^{n}$.

A2. $A=\left(a_{i j}\right)$ and $C=\left(C_{i j}\right)$ are both irreducibly diagonally dominant with positive diagonal and non-positive off-diagonal entries, i.e., they are irreducible and satisfy, for $i, j=1,2, \ldots, n$,

$$
a_{i i}>0, c_{i i}>0, a_{i j} \leq 0, c_{i j} \leq 0, i \neq j, a_{i i} \geq \sum_{j \neq i}\left|a_{i j}\right|, c_{i i} \geq \sum_{j \neq i}\left|c_{i j}\right|,
$$


and there exist at least $k$ and $l$ such that $a_{k k}>\sum_{j \neq k}\left|a_{k j}\right|$ and $c_{l l}>$ $\sum_{j \neq l}\left|c_{l j}\right|$. Under these conditions, $A$ and $C$ are both $M$-matrices by [27]. A3. Problem 1 has a solution.

Clearly, Problem 1 can be solved by many existing generic methods for complementarity problems such as the semi-smooth Newton method [17], the smooth methods [22], the active-set Newton methods [7] and the interior-point method [25], just to name a few. However, Problem 1 differs from a general LCP because of Assumptions A1 and A2. Therefore, it would be ideal if these properties can be exploited in the design of a numerical method for it. Recently, penalty methods have been used extensively for solving various linear and nonlinear complementarity problems, particularly those arising in financial engineering $[23,26,33,31,15,16,29,21,32,30]$. Most of these methods are based on power penalty terms to penalize the components of an approximation which are infeasible. The power penalty methods have the merit that they have exponential convergence rates. However, the power penalty methods are exterior in the sense that the constraints (3) may not be strictly satisfied by the solutions from the methods. An empirical interior penalty method in infinite-dimensions for option pricing problems is proposed in [23,26] and used by various authors such as [14]. A convergence analysis for a similar method in an infinite-dimensional functional space setting is conducted in [37]. However, to the best of our knowledge, there are no systematic studies on interior methods for large-scale LCPs arising particularly in option valuation or obstacle problems in financial and classic engineering, though such methods have been studied extensively for solving general linear and nonlinear programming problems [12].

Motivated by the penalty method in $[23,26]$ and conventional interior methods in constrained optimization, we propose in this work an interior method for (5) and study its mathematical properties such as its solvability and convergence. In this approach, we approximate (5) by a nonlinear equation system containing a penalty or barrier term to prevent approximate solutions from becoming infeasible. This penalty term is in fact the first derivative of a wellknown logarithmic barrier function often used in constrained optimization. Unlike most of the existing interior methods for (5) (cf., for example, $[25,10]$ ), our method does not require any auxiliary functions such as potential or merit functions which often result in a global optimization problem even when the original problem is monotone or strictly monotone $[17,36]$. We will establish a convergence theory for the approximate solution. The rest of this paper is organised as follows.

In the next section we propose the interior penalty approach and prove that the penalty equation has a solution under Assumptions A1 and A2. In Section 3, we establish a convergence theory for the approach. In Section 4 we propose a smooth Newton's method. Numerical experimental results using three test problems are presented in Section 5 to demonstrate the theoretical rates of convergence and usefulness of the methods. 
Finally, we comment that although our focus of this work is on LCPs in financial engineering, the developed method is also applicable to LCPs arising from classic engineering satisfying Assumptions A1 and A2.

\section{The interior method}

Without loss of generality, we assume that $d=0$ in (5). A simple transformation will transform the case that $d \neq 0$ into that with $d=0$.

Interior methods have been used very successfully for solving constrained optimization problems [12]. These methods have the merit that a solution from such a method satisfies the constraints strictly. When $A$ in (2) is symmetric, $C=I$ and $d=0$, where $I$ denotes the $n \times n$ identity matrix, in (5), it is trivial to verify that (5) is the set of the KKT conditions of the following quadratic programming problem:

$$
\min _{x \in \mathbb{R}^{n}}\left(\frac{1}{2} x^{\top} A x-b^{\top} x\right), \quad \text { subject to } \quad x \leq 0 .
$$

A popular interior method for (6) is based on the use of a logarithmic barrier function whose value becomes infinite when the decision variable approaches the boundary of the infeasible region. Mathematically, (6) is approximated by the following unconstrained problem:

$$
\min _{x \in \mathbb{R}^{n}}\left(\frac{1}{2} x^{\top} A x-b^{\top} x-\mu \sum_{i=1}^{n} \ln \left(-x_{i}\right)\right),
$$

where $\mu>0$ is a parameter and $x=\left(x_{1}, x_{2}, \ldots, x_{n}\right)^{\top}$. Clearly, we look for solutions $x_{\mu}$ satisfying $x_{\mu}<0$ and expect that $x_{\mu} \rightarrow x$, the solution to (6), as $\mu \rightarrow+0$. Due to the barrier function, we expect that solution trajectory generated by an algorithm such as the Newton method will never escape from the feasible region if the initial guess is feasible. A convergence theory when $\mu \rightarrow+0$ for this interior method under certain conditions can be found in [12].

Before further discussion, we first define some notation to be used in the rest of this paper. For any $y \in \mathbb{R}^{n}$, we let $y \cdot / z=\left(y_{1} / z_{1}, \ldots, y_{n} / z_{n}\right)^{\top}$, i.e., ./, used in Matlab, denotes the Hadamard element by element division of two matrices of the same size with the exception that $y \cdot / z=\left(y / z_{1}, \ldots, y / z_{n}\right)^{\top}$ if $y$ is a scalar. For any two functions $f$ and $g$, we will use $f(x) \leq \mathcal{O}(g(x))$ to represent the relationship that there is a positive constant $C$ such that $f(x) \leq C g(x)$ as $x$ approaches a fixed value or infinity. Using the above notation, we write the first-order optimality condition for the solutions to (7) as

$$
A x_{\mu}-\mu \cdot / x_{\mu}=b,
$$

where $x_{\mu}=\left(x_{\mu, 1}, x_{\mu, 2}, \ldots, x_{\mu, n}\right)^{\top} \in \mathbb{R}^{n}$. Thus, any negative solution to (8) is a minimum point of (7) when $A$ is symmetric. 
For most of practical problems arising in financial engineering such as the discretized system of (2)-(4) using any of $[28,9,19,20], A$ is usually unsymmetric. In this case, the first-order optimality conditions of (6) are not of the form (5) even when $C=I$ and $d=0$. In fact, (5) may not be the KKT conditions of a constrained optimization problem.

Motivated by (8), we propose to approximate (5) by the following nonlinear equation:

$$
A x_{\mu}-\mu \cdot /\left(C x_{\mu}\right)=b,
$$

where $\mu>0$ is a parameter. Clearly, our main concerns are whether (9) has a solution satisfying $C x_{\mu}<0$ and if so, whether such a solution converges to the solution $x$ to (5) as $\mu \rightarrow+0$. Before considering the convergence of this approach, we first show that there exists a solution to (9). We start this discussion with following lemma.

Lemma 1 For any given $\mu>0$, if $x_{\mu}$ is a solution to (9) such that $C x_{\mu}<0$, then $x_{\mu}$ satisfies

$$
\left\|x_{\mu}\right\| \leq M
$$

where $M>0$ is a constant, independent of $\mu$.

Proof We let $M$ denote a generic positive constant, independent of $\mu$, and use $\|\cdot\|_{\infty}$ to denote the $l^{\infty}$-norm on $\mathbb{R}^{n}$. For a positive constant $\alpha$ independent of $\mu$, we consider the following two cases.

Case 1. $\left\|C x_{\mu}\right\|_{\infty} \geq \alpha \mu$.

Left-multiplying both sides of (9) by $x_{\mu}^{\top}$ we have

$$
x_{\mu}^{\top} A x_{\mu}-x_{\mu}^{\top}(\mu \cdot /(C x))=x_{\mu}^{\top} b .
$$

Thus, reorganising this equation and using Assumption A1, we have

$$
a_{0}\left\|x_{\mu}\right\|^{2} \leq x_{\mu}^{\top} A x_{\mu} \leq \mu\left\|1 . /\left(C x_{\mu}\right)\right\|_{\infty}\left\|x_{\mu}^{\top}\right\|+\|b\| \cdot\left\|x_{\mu}\right\| \leq\left(\frac{1}{\alpha}+\|b\|\right)\left\|x_{\mu}\right\| .
$$

From this we have (10).

Case 2. $\left\|C x_{\mu}\right\|_{\infty}<\alpha \mu$.

We first note that $C$ is an $M$-matrix by Assumption A2 and thus $C^{-1}$ is positive. From the positiveness of $C^{-1}$ and $C x_{\mu}<0$ we have $x_{\mu}<0$.

Since $\left\|C x_{\mu}\right\|_{\infty}<\alpha \mu$ and $C x_{\mu}<0$, we have

$$
0<-C x_{\mu} \leq \alpha \mu e, \quad \text { or, } \quad 0>C x_{\mu} \geq-\alpha \mu e,
$$

where $e=(1,1, \ldots, 1)^{\top} \in \mathbb{R}^{n}$. Using the positiveness of $C^{-1}$ we have from the above $0>x_{\mu} \geq-\alpha \mu C^{-1} e$. Therefore, we see that (10) also holds true for this case.

Combining the above two cases we have (10).

Using this lemma, we prove that (9) has a solution in the following theorem.

Theorem 1 For any $\mu>0$, there exists a solution $x_{\mu}$ to (9) satisfying $C x_{\mu}<$ 0 . 
Proof We show that (9) has a solution in a bounded region $S=\left\{x \in \mathbb{R}^{n}\right.$ : $\left.-\varepsilon^{-1} e<C x<-\delta e\right\}$, where $e=(1, \ldots, 1)^{\top}$ and $\varepsilon$ and $\delta$ are (sufficiently small) positive constants. Let $F(x):=A x-\mu$. $/(C x)$. Clearly, $F: \bar{S} \subset \mathbb{R}^{n} \mapsto \mathbb{R}^{n}$ is continuous. To prove this theorem, it suffices to verify that the conditions of Kronecker Theorem [24, p.161] that $b$ is not in the image of the boundary of $S$ under the mapping $F$ and the degree of $\nabla F$ is non-zero are satisfied. More specifically, we will show that $b \notin F(\partial S)$ when both $\varepsilon>0$ and $\delta>0$ are sufficiently small and $\nabla F$ is non-singular, where $\partial S$ denotes the boundary of $S$.

To prove the former, we assume that $b \in F(\partial S)$, that is, there exists an $x \in \partial S$ such that $F(x)=b$. Then, we show this is not possible when both $\delta$ and $\varepsilon$ are sufficiently small in the following two cases:

Case 1. Suppose there is a $k \in\{1, \ldots, n\}$ such that the $k$ th component of $C x$ is $(C x)_{k}=-\delta$. Then, we have

$$
F_{k}(x)=(A x)_{k}-\frac{\mu}{(C x)_{k}}=a_{k k} x_{k}+\sum_{j \neq k} a_{k j} x_{j}+\frac{\mu}{\delta} .
$$

Note that $F_{k}(x)=b_{k}$. Since $C$ is an $M$-matrix, $C^{-1}$ is non-negative and thus $C x<0$ implies $x<0$. Therefore, from (11) we see that $a_{k k} x_{k} \leq-\alpha(\mu / \delta)$ for a positive constant $\alpha$, independent of $\mu$ and $\delta$, because $\sum_{j \neq k} a_{k j} x_{j} \geq 0$, where. Therefore, $x_{k} \rightarrow-\infty$ as $\delta \rightarrow+0$. This violates (10) in Lemma 1, and thus we conclude that when $\delta>0$ is sufficiently small, $b \notin F(\partial S)$ with $C x_{k}=-\delta$ for a feasible $k$.

Case 2. We now consider the case that $x$ is on $\partial S$ such that at least one component of $C x$ is equal to $(-\beta / \varepsilon)$ for a positive constant $\beta$, independent of $\varepsilon$ and $\mu$, i.e., $(C x)_{k}=-\beta \varepsilon^{-1}$ for a feasible index $k$. In this case we have $x=C^{-1}(\cdot, \cdot, \ldots, \underbrace{-\beta \varepsilon^{-1}}_{k \mathrm{th}}, \ldots, \cdot)^{\top}$. Since $C$ is an $M$-matrix, $C^{-1}:=\left(\bar{c}_{i j}\right)$ is nonnegative. Also, there must be at least one index $l$ such that $\bar{c}_{k l} \neq 0$, as otherwise, $C^{-1}$ is singular. Note also that $x<0$ as reasoned in the proof of Case 1 above. Thus, combining $\bar{c}_{k l} \neq 0$, non-negativity of $C$ and $x<0$ we have $x_{l} \leq-\bar{c}_{k l} \beta \varepsilon^{-1} \rightarrow-\infty$ as $\varepsilon \rightarrow+0$. Therefore, Lemma 1 is also violated by this $x$ and thus we see that $b \notin F(\partial S)$ with $(C x)_{k}=-\beta \varepsilon^{-1}$.

Combining the above two cases we see that when $\varepsilon>0$ and $\delta>0$ are both sufficiently small, $b \notin F(\partial S)$. Furthermore, it is easy to see that the Jacobian matrix of $F$ is $\nabla F(x)=A+\mu D(x) C$, where

$$
D(x)=\operatorname{diag}(1 . /(C x) . /(C x))=\operatorname{diag}\left(\frac{1}{(C x)_{1}^{2}}, \ldots, \frac{1}{(C x)_{n}^{2}}\right) .
$$

Note that

$$
D(x) C=\left(\begin{array}{cccc}
d_{1} c_{11} & d_{1} c_{12} & \cdots & d_{1} c_{1 n} \\
d_{2} c_{21} & d_{2} c_{22} & \cdots & d_{2} c_{2 n} \\
\vdots & \vdots & \ddots & \vdots \\
d_{n} c_{n 1} & d_{n} c_{n 2} & \cdots & d_{n} c_{n n}
\end{array}\right)
$$


where $d_{i}=(C x)_{i}^{-2}$. Since $C$ is irreducibly diagonally dominant with $c_{i i}>0$ and $c_{i j} \leq 0$ when $i \neq j$ by Assumption A2, from (13) we see that $D C$ is also irreducibly diagonally dominant. Therefore, it is an $M$-matrix, and so $\nabla F$ is non-singular when $C x<0$. The non-singularity implies that the degree of $F$, defined as $\operatorname{sign}(\operatorname{det}(\nabla F))$, at any point of $S$ is non-zero. Therefore, by the Kronecker Theorem, $F(x)=b$ has a solution in $S$ [24, p.161].

Thus we have proved the theorem.

\section{Convergence}

In the previous section we showed that the penalty equation (9) has a solution satisfying $C x_{\mu}<0$. We now prove that $x_{\mu}$ converges to that of (5) as $\mu \rightarrow+0$. We start this discussion with the following theorem.

Theorem 2 Let $\mu>0$ be a constant. Any solution to (9) such that $C x_{\mu}<0$ satisfies the $\operatorname{LCP}$ (5) with $d=0$ as $\mu \rightarrow+0$.

Proof When $C x_{\mu}<0$, from (9) we have

$$
A x_{\mu}-b=\mu . /\left(C x_{\mu}\right)<0 .
$$

Left-multiplying both sides of $(9)$ by $\left(C x_{\mu}\right)^{\top}$ and rearranging the resulting equation give

$$
\begin{aligned}
\left(C x_{\mu}\right)^{\top}\left(A x_{\mu}-b\right) & =\mu\left(C x_{\mu}\right)^{\top}\left(1 . /\left(C x_{\mu}\right)\right) \\
& =\mu\left((C x)_{1}, \ldots,(C x)_{n}\right)\left(\frac{1}{(C x)_{1}}, \ldots, \frac{1}{(C x)_{n}}\right)^{\top}=\mu n .
\end{aligned}
$$

Therefore, when $\mu \rightarrow+0, x_{\mu}$ satisfies (5).

In Theorem 2 we have established the convergence of the solution of (9) to that of (5) without explicitly using Assumptions A1 and A2, though they are used in Theorem 1. In our computation, we use (14) as one of the stopping criteria for the iterative process. However, this theorem does not provide any rate of convergence of $x_{\mu}$. If, in addition to Assumptions A1 and A2, we further assume that $C^{\top} A$ is positive-definite, we are able to show that both Problem 1 and (9) have a unique solution and that the solution to (9) converges to that of Problem 1 at the rate of $\mathcal{O}\left(\mu^{1 / 2}\right)$ as $\mu \rightarrow+0$. These results are given in the following theorem.

Theorem 3 Let Assumptions A1 and A2 be fulfilled. In addition, we assume that $C^{\top} A$ is also positive-definite, i.e., there exists a constant $a_{1}>0$ such that $x^{\top} C^{\top} A x \geq a_{1}\|x\|^{2}$ for any $x \in \mathbb{R}^{n}$. Let $x^{*}$ and $x_{\mu}$ such that $C x_{\mu}<0$ be solutions to Problem 1 and (9) respectively. Then, $x^{*}$ and $x_{\mu}$ are unique for any $\mu>0$ and satisfy

$$
\left\|x^{*}-x_{\mu}\right\| \leq \sqrt{\frac{n \mu}{a_{1}}} .
$$


Proof Suppose both $x^{*}$ and $y^{*}$ satisfy (5) (with $d=0$ ). From $C y^{*} \leq 0$ and $A x^{*} \leq b$ we have

$$
\left(C y^{*}\right)^{\top} A x^{*} \geq\left(C y^{*}\right)^{\top} b .
$$

From the equation in (5) we have

$$
\left(C x^{*}\right)^{\top}\left(A x^{*}-b\right)=0 .
$$

Combining this equality and (16) we get

$$
\left(C\left(y^{*}-x^{*}\right)\right)^{\top} A x^{*} \geq\left(C\left(y^{*}-x^{*}\right)\right)^{\top} b .
$$

By symmetry, we also have

$$
\left(C\left(x^{*}-y^{*}\right)\right)^{\top} A y^{*} \geq\left(C\left(x^{*}-y^{*}\right)\right)^{\top} b
$$

Adding up both sides of (18) and (19) gives

$$
\left(C\left(y^{*}-x^{*}\right)\right)^{\top} A\left(x^{*}-y^{*}\right) \geq 0 \quad \text { or } \quad\left(x^{*}-y^{*}\right)^{\top} C^{\top} A\left(x^{*}-y^{*}\right) \leq 0 .
$$

Therefore, using the positive definiteness of $C^{\top} A$ we obtain $x^{*}=y^{*}$.

The proof of the uniqueness of $x_{\mu}$ is rather trivial and we omit it.

We now prove (15). Left-multiplying (9) by $\left(C\left(x_{\mu}-x^{*}\right)\right)^{\top}$, we have

$$
\left(C\left(x_{\mu}-x^{*}\right)\right)^{\top} A x_{\mu}-\left(C\left(x_{\mu}-x^{*}\right)\right)^{\top}\left(\mu \cdot /\left(C x_{\mu}\right)\right)=\left(C\left(x_{\mu}-x^{*}\right)\right)^{\top} b .
$$

Since $x^{*}$ is a solution to Problem 1 and $C x_{\mu}<0$, left-multiplying both sides of $(5)$ by $\left(C x_{\mu}\right)^{\top}$ gives

$$
\left(C x_{\mu}\right)^{\top} A x^{*} \geq\left(C x_{\mu}\right)^{\top} b .
$$

Combining the above inequality with (17) we have

$$
\left(C\left(x_{\mu}-x^{*}\right)\right)^{\top} A x^{*} \geq\left(C\left(x_{\mu}-x^{*}\right)\right)^{\top} b .
$$

Now, subtracting both sides of (20) from the corresponding sides of (21) yields

$$
\left(C\left(x_{\mu}-x^{*}\right)\right)^{\top} A\left(x^{*}-x_{\mu}\right)+\mu\left(C\left(x_{\mu}-x^{*}\right)\right)^{\top}\left(1 . /\left(C x_{\mu}\right)\right) \geq 0 .
$$

From this inequality we have

$$
\begin{aligned}
\left(x^{*}-x_{\mu}\right)^{\top}\left(C^{\top} A\right)\left(x^{*}-x_{\mu}\right) & \leq \mu\left(C\left(x_{\mu}-x^{*}\right)\right)^{\top}\left(1 . /\left(C x_{\mu}\right)\right) \\
& =\mu\left(n-\left(C x^{*}\right)^{\top}\left(1 . /\left(C x_{\mu}\right)\right)\right) \leq \mu n,
\end{aligned}
$$

since $\left.\left(C x^{*}\right)^{\top}\left(1 . /\left(C x_{\mu}\right)\right)\right) \geq 0$. Finally, using the positive definiteness of $C^{\top} A$ we have from the above estimate

$$
a_{1}\left\|x^{*}-x_{\mu}\right\|^{2} \leq\left(x^{*}-x_{\mu}\right)^{\top} C^{\top} A\left(x^{*}-x_{\mu}\right) \leq \mu n,
$$

from which we have (15). 
We comment that while Theorem 3 provides an upper bound for the error in $x_{\mu}$, this bound may not be sharp. In fact, from (22) we see that if $\left\|1 . /\left(C x_{\mu}\right)\right\|$ is uniformly bounded, then from the first inequality in (22) we can easily deduce $\left\|x_{\mu}-x^{*}\right\| \leq \frac{\mu}{a_{1}}\|C\| \cdot\left\|1 . /\left(C x_{\mu}\right)\right\|$. However, in practice, some components of $C x_{\mu}$ converge to zero as $\mu \rightarrow+0$, and thus $\left\|1 . /\left(C x_{\mu}\right)\right\|$ is usually unbounded as $\mu \rightarrow+0$. Nevertheless, in our computation we have found that the computed rates of convergence are normally higher than the theoretical one given in (15).

We also comment that Theorem 3 contains the conventional type of LCPs with $C=I$ as a special case in which $C^{\top} A=A$ is positive-definite by Assumption A1. In general, even the positive-definiteness of $C^{\top} A$ is not satisfied, numerical solutions from this approach still converge to the exact solution at a rate no lower than that in (15). We will demonstrate this numerically using one test example later in this work.

\section{Newton's method for (9)}

Equation (9) is nonlinear and smooth in the interior of the convex set $\{x \in$ $\left.\mathbb{R}^{n}: C x \leq 0\right\}$ and a smooth Newton method can be used for it. Since we only look for negative solution to (9) the main consideration in designing a Newton algorithm is to avoid an iterate to become infeasible, or 'overshot', due to the linearization in the Newton method. We propose the following Newton algorithm for (9).

\section{Algorithm Newton}

Step 1. Choose $\mu>0, \varepsilon>0$ and $\delta>0$ sufficiently small and an initial guess $x_{0} \in \mathbb{R}^{n}$ such that $C x^{0}<0$. Let $k:=0$.

Step 2. Solve the following linear system for $p^{k}$ :

$$
\left[A+\mu D\left(x^{k}\right) C\right] p^{k}=-\left(A x^{k}-\mu \cdot /\left(C x^{k}\right)-b\right),
$$

where $D$ is defined in (12).

Step 3. Set $x^{k+1}=C^{-1}\left(\min \left\{C\left(x^{k}+p^{k}\right),-\delta e\right\}\right)$, where $e=(1,1, \ldots, 1)^{\top} \in \mathbb{R}^{n}$.

Step 4. If

$$
\max \left\{\left\|x^{k+1}-x^{k}\right\|,\left|\left(C x^{k+1}\right)^{\top}\left(A x^{k+1}-b\right)-\mu n\right|\right\} \leq \varepsilon,
$$

then stop. Otherwise, set $k:=k+1$ and go to Step 2 .

We comment that in Step 3 of Algorithm Newton, we use an upper bound to force an approximate solution to become strictly feasible when overshooting occurs in a Newton iteration due to the linear approximation. Since the solution $x_{\mu}$ to $(9)$ satisfies $C x_{\mu}<0$, when an iterate in Newton's algorithm becomes infeasible due to the linear approximation of the barrier function, the usual practice is to use the ' 0.99 ' rule [12], i.e., use a damping parameter to reduce the step length of the new iterate so that it is taken as $99 \%$ of the distance to the finite boundary of the feasible region. However, this rule does not work well for solving the type of LCP here from our computation experience. 
The reason may be that many of the constraints in (5) will become active and ' 0.99 ' rule restricts the rate of convergence of approximate solutions to the boundary of the feasible region.

In view of the numerical solution of (23), from the last part of the proof of Theorem 1 we see that the system matrix $A+\mu D\left(x^{k}\right) C$ of $(23)$ is an $M$ matrix, and thus efficient iterative methods such as the preconditioned CGS or BiCGSTAB can be used for solving (23). Note that $C^{-1}$ is used in Step 3 of the above algorithm which incurs addition computational costs. In practice, we need to calculate the LU decomposition of $C$ only once. Also, for many practical problems $C$ is either an identity matrix or an upper/lower triangular matrix as will be seen in the next section.

\section{Numerical experiments}

In this section we present some numerical experimental results to demonstrate the efficiency, rates of convergence and usefulness of the numerical method presented and analysed above. Two test problems with un-symmetric system matrices and an American option pricing problem are chosen. In our numerical experiments, the parameters $\varepsilon$ and $\delta$ in Step 1 of Algorithm Newton are chosen to be $\varepsilon=10^{-10}$ and $\delta=10^{-12}$. All experiments were performed in double precision under Matlab environment.

Test 1. The LCP (1) in two dimensions with the following operators and functions:

$$
\begin{aligned}
L_{1} & =-\left(\frac{\partial^{2}}{\partial x^{2}}+\frac{\partial^{2}}{\partial y^{2}}\right)+\frac{\partial}{\partial x}+\frac{\partial}{\partial y}, L_{2}=I, \\
f & =5\left[6 x y\left(2-x^{2}-y^{2}\right)+\left(1-3 x^{2}\right)\left(y-y^{3}\right)+\left(1-3 y^{2}\right)\left(x-x^{3}\right)\right], \\
u^{*} & =0.3+|x-0.5|+|y-0.5|
\end{aligned}
$$

for $(x, y) \in \Omega:=(0,1)^{2}$ satisfying $u=0$ on the boundary of $\Omega$, where $I$ denotes the identity operator. The exact solution to the unconstrained problem $L_{1} u=f$ is $u_{\text {unc }}=5\left(x-x^{3}\right)\left(y-y^{3}\right)$.

This is a conventional LCP in which $u^{*}$ can be viewed as an obstacle for the solution. To solve this infinite-dimensional LCP numerically, we first choose a mesh for $\Omega$ with $(N+1) \times(N+1)$ nodes $\left(x_{i}, y_{j}\right)=(i / N, j / N)$ for $i, j=0,1, \ldots, N$, where $N$ denotes a positive integer. For each of the mesh nodes $\left(x_{i}, y_{j}\right), i, j=1,2, \ldots, N$, we use the conventional central difference scheme and backward difference scheme to approximate respectively the 2nd-order and the 1st-order differential operators in $L_{1} u[20,19,21]$. All other terms are approximated by their respective nodal values at $\left(x_{i}, y_{j}\right)$. This discretization method results in an LCP of the form $(5)$ in $\mathbb{R}^{(N-1)^{2}}$ with $A$ an un-symmetric penta-diagonal system matrix $A$. In this case, it is easy to verify that $C^{\top} A=A$ is positive-definite. In our computation, we choose $N=100$ and the initial guesses for all the penalty equation are chosen to be zero. 


\begin{tabular}{|c|ccccccc|}
\hline$\mu=\frac{10^{-2}}{2^{i}}$ & $i=0$ & $i=1$ & $i=2$ & $i=3$ & $i=4$ & $i=5$ & $i=6$ \\
\hline Error & $1.26 \mathrm{e}-1$ & $6.69 \mathrm{e}-2$ & $3.54 \mathrm{e}-3$ & $1.87 \mathrm{e}-2$ & $9.91 \mathrm{e}-3$ & $5.25 \mathrm{e}-3$ & $2.79 \mathrm{e}-3$ \\
Ratio & - & 1.88 & 1.89 & 1.89 & 1.89 & 1.89 & 1.88 \\
\hline \hline & $i=7$ & $i=8$ & $i=9$ & $i=10$ & $i=11$ & $i=12$ & $i=13$ \\
\hline & $1.49 \mathrm{e}-3$ & $7.95 \mathrm{e}-4$ & $4.24 \mathrm{e}-4$ & $2.25 \mathrm{e}-4$ & $1.19 \mathrm{e}-4$ & $6.24 \mathrm{e}-5$ & $3.23 \mathrm{e}-5$ \\
& 1.88 & 1.87 & 1.88 & 1.88 & 1.89 & 1.91 & 1.93 \\
\hline
\end{tabular}

Table 1 Computed rates of convergence in $\mu$ for Test 1 .

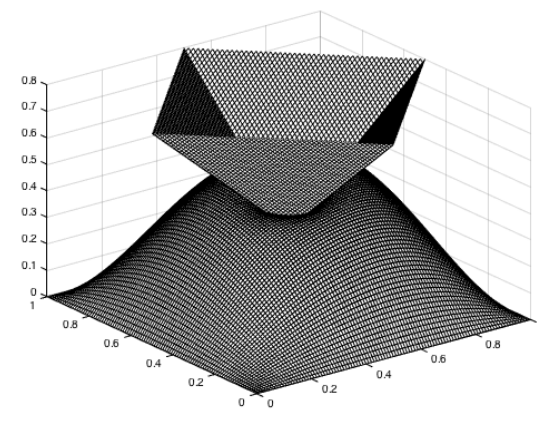

(a) $u$ and $u^{*}$

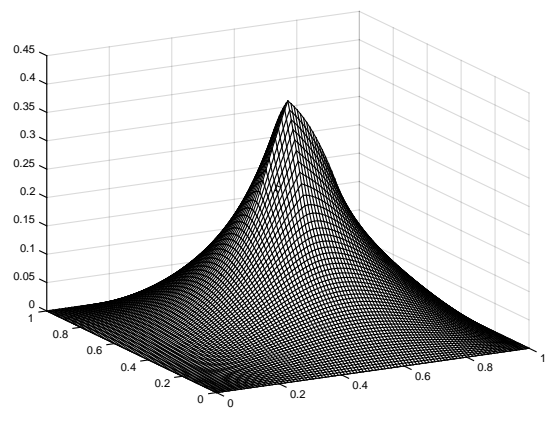

(b) $u_{\mathrm{unc}}-u$

Fig. 1 Computed $u, u^{*}$ and $u_{\mathrm{unc}}-u$ for Test 1 .

To compute the rates of convergence of the interior method, we solve the finite-dimensional LCP using $\mu=10^{-10}$ and use the numerical solution as the 'exact' or reference solution. The problem is then solved for $\mu=10^{-2} / 2^{j}$ for $j=0,1, \ldots, 13$ and the $l_{2}$-norms of the errors between the numerical and reference solutions are calculated. Table 1 contains the computed errors and the ratios of errors from two consecutive values of $\mu$. Theoretically, the ratios are no less than $\sqrt{2}$ by (15). From Table 1 we see that the rate of convergence for this test problem is about $\mathcal{O}\left(\mu^{\log _{2} 1.9}\right) \approx \mathcal{O}\left(\mu^{0.925}\right)$. The numerical solution $u$, upper bound $u^{*}$ and the difference between the unconstrained and numerical solutions, $u_{\mathrm{unc}}-u$, are plotted in Figure 1 from which we see that the numerical solution is qualitatively excellent. In our numerical experiments, the initial guess in Step 1 of Algorithm Newton is $x_{0}=0$ for all values of $\mu$ and the mean of the numbers of Newton's iterations for all the values of $\mu$ is 33.3 with the standard deviation 7.3, indicating the numerical method is robust with respect to $\mu$.

Test 2. The LCP (1) in two dimensions with the following operators and functions:

$$
\begin{aligned}
L_{1} & =-\left(\frac{\partial^{2}}{\partial x^{2}}+\frac{\partial^{2}}{\partial y^{2}}\right)+\frac{\partial}{\partial x}+\frac{\partial}{\partial y}, L_{2}=\frac{\partial}{\partial x}+\frac{\partial}{\partial y}, \\
f & =5\left[6 x y\left(2-x^{2}-y^{2}\right)+\left(1-3 x^{2}\right)\left(y-y^{3}\right)+\left(1-3 y^{2}\right)\left(x-x^{3}\right)\right], u^{*}=1
\end{aligned}
$$




\begin{tabular}{|c|ccccccc|}
\hline$\mu=\frac{10^{-2}}{2^{i}}$ & $i=0$ & $i=1$ & $i=2$ & $i=3$ & $i=4$ & $i=5$ & $i=6$ \\
\hline Error & $6.97 \mathrm{e}-2$ & $3.52 \mathrm{e}-2$ & $1.77 \mathrm{e}-2$ & $8.89 \mathrm{e}-3$ & $4.46 \mathrm{e}-3$ & $2.24 \mathrm{e}-3$ & $1.12 \mathrm{e}-3$ \\
Ratio & - & 1.98 & 1.99 & 1.99 & 1.99 & 1.99 & 1.99 \\
\hline \hline & $i=7$ & $i=8$ & $i=9$ & $i=10$ & $i=11$ & $i=12$ & $i=13$ \\
\hline & $5.64 \mathrm{e}-4$ & $2.83 \mathrm{e}-4$ & $1.42 \mathrm{e}-4$ & $7.13 \mathrm{e}-5$ & $3.57 \mathrm{e}-5$ & $1.79 \mathrm{e}-5$ & $8.98 \mathrm{e}-5$ \\
& 1.99 & 1.99 & 1.99 & 1.99 & 2.00 & 2.00 & 2.00 \\
\hline
\end{tabular}

Table 2 Computed rates of convergence in $\mu$ for Test 2 .

for $(x, y) \in \Omega:=(0,1)^{2}$ satisfying $u=0$ on the boundary of $\Omega$. The exact solution to the unconstrained problem $L_{1} u=f$ is $u_{\mathrm{unc}}=5\left(x-x^{3}\right)\left(y-y^{3}\right)$.

Clearly, the difference between this test and Test 1 is that Test 1 has an upper bound on the solution while Test 2 has one on the the sum of the two first derivatives of the solution. We use the same mesh as used in Test 1 with $(N+1)^{2}$ mesh nodes to solve this problem. The discretized form of $L_{1}$ is the same as that in Test 1 and the resulting LCP is in $\mathbb{R}^{n}$ with $n=(N-1)^{2}$. We also use the two-point backward finite difference scheme to discretize $L_{2}$ so that the system matrix $C=\left(c_{i j}\right)$ in (5) has only 3 non-zero diagonals, i.e., $c_{i i}>0$ for $i=1,2, \ldots, n, c_{i-1, i} \leq 0$ for $i=2,3 \ldots, n, c_{(i-N-1) j} \leq 0$ for $i=N, \ldots, n$, and $c_{i j}=0$ for all other feasible $(i, j)$.

As in Test 1, we use the numerical solution from $\mu=10^{-10}$ as a reference solution for calculating computational errors. The problem is then solved for $\mu=10^{-2} / 2^{j}$ with $j=0,1, \ldots, 13$ and the $l_{2}$-norms of the errors between the numerical and reference solutions are calculated. The computed errors, along with the ratios of those from two consecutive values of $\mu$, are listed in Table 2 . From the ratios we see that the computed rates of convergence for this test are of order $\mathcal{O}(\mu)$, though in this case $C^{\top} A$ may not be positive-definite. For all the chosen values of $\mu$, the initial guess is $x_{0}=0$ and the average number of Newton iterations is 34.3 with the standard deviation 5.08 which are in line with that for Test 1 . To further demonstrate the numerical method, we plot, in Figure 2, the numerical solution of $L_{2} u$ along with the upper bound $u^{*}$ and the difference between the unconstrained and constrained the numerical solutions. From the figure we again see that numerical solution is qualitatively excellent.

Test 3. American put option valuation problem (2)-(4) defined on $(0,100) \times$ $[0,1)$ with the parameters $\sigma=0.4, r=0.03$ and strike price $K=50$. The lower bound $V^{*}$ and boundary and payoff conditions are

$V^{*}(S)=\max \{0, K-S\}, V(0, t)=K, V(100, t)=0, V(S, t=T)=V^{*}(S)$.

To solve this problem numerically, we choose an $(M+1) \times(N+1)$ uniform mesh with nodes $\left(S_{i}, t_{j}\right)$ for $i=0, \ldots, M$ and $j=0,1, \ldots, N$, where $M$ and $N$ denote two positive integers. The infinite-dimensional LCP (2)-(4) is then discretized using the fitted finite volume method in $[28,34]$ and at each time step $j$, we solve an LCP in $\mathbb{R}^{M-1}$. (For an analysis of the discretization method, 


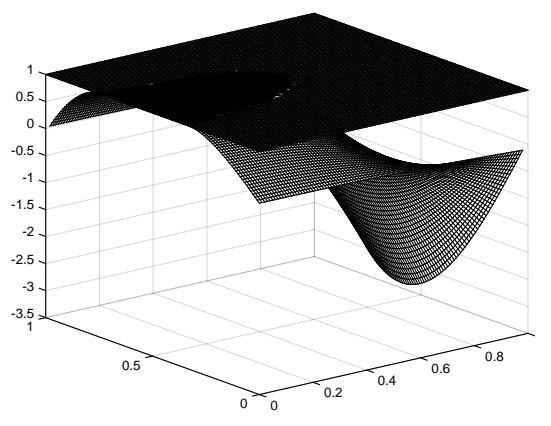

(a) $L_{2} u$ and $u^{*}$

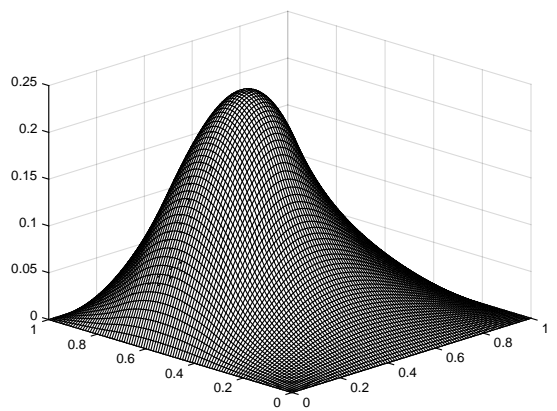

(b) $u_{\text {unc }}-u$

Fig. 2 Computed $L_{2} u, u^{*}$ and $u_{\mathrm{unc}}-u$ for Test 2 .

\begin{tabular}{|c|ccccccc|}
\hline$\mu=\frac{10^{-4}}{2^{i}}$ & $i=0$ & $i=1$ & $i=2$ & $i=3$ & $i=4$ & $i=5$ & $i=6$ \\
\hline Error & $1.400 \mathrm{e}-1$ & $9.259 \mathrm{e}-2$ & $6.13 \mathrm{e}-2$ & $4.07 \mathrm{e}-2$ & $2.70 \mathrm{e}-2$ & $1.80 \mathrm{e}-2$ & $1.19 \mathrm{e}-2$ \\
Ratio & - & 1.51 & 1.51 & 1.51 & 1.51 & 1.50 & 1.50 \\
\hline \hline & $i=7$ & $i=8$ & $i=9$ & $i=10$ & $i=11$ & $i=12$ & $i=13$ \\
\hline & $7.94 \mathrm{e}-3$ & $5.28 \mathrm{e}-3$ & $3.51 \mathrm{e}-3$ & $2.23 \mathrm{e}-3$ & $1.54 \mathrm{e}-3$ & $1.01 \mathrm{e}-3$ & $6.58 \mathrm{e}-4$ \\
& 1.50 & 1.50 & 1.51 & 1.51 & 1.51 & 1.52 & 1.53 \\
\hline
\end{tabular}

Table 3 Computed rates of convergence in $\mu$ for Test 3 .

we refer to [1].) For most of the numerical experiments, we choose $M=N=$ 100. Clearly, in this case $C$ is the identity matrix and it is easy to verify that at each time step, the system matrix $A$ is un-symmetric and positive-definite. Since the exact solution to this LCP is unknown, we use the numerical solution with $\mu=10^{-10}$ as the 'exact' solution. The discretized American option pricing problem is solved for $\mu=10^{-4} / 2^{j}$ for $j=0,1, \ldots, 13$. The computed errors in the last time point $t=0$ and their ratios are listed in Table 3. From the table we see that the computed rates of convergence are roughly $\mathcal{O}\left(\mu^{0.59}\right)$, close to the theoretical result $\mathcal{O}\left(\mu^{0.5}\right)$. At each time step $j=2,3, \ldots, M$, we use the numerical solution at $j-1$ as the initial guess for the Newton's method, while for $j=1$ we use $\left(V^{*}\left(S_{1}\right)+\delta, \ldots, V^{*}\left(S_{M-1}\right)+\delta\right)^{\top}$ as the initial guess. The mean and standard deviation of the numbers of Newton iterations for all $\mu$ and all time steps are 5.0 and 3.7 respectively, indicating that the method is efficient and robust.

To further demonstrate the quality of the results from our numerical method, we plot in Figure 3 the computed value function $V$ and its derivatives $\Delta:=$ $V_{S}(S, t)$ and $\Gamma:=V_{S S}(S, t)$ which are often used in hedging strategies. From the figure we see that the numerical results are qualitatively excellent. Also, the optimal exercise curve is clearly visible in Figures 3 (b) and (c). This curve is mathematically called a free boundary which is part of the solution. To further demonstrate this curve, we recompute the solution using $M=500$ and $N=100$. The optimal exercise curve is estimated using the computed $\Delta$ and 


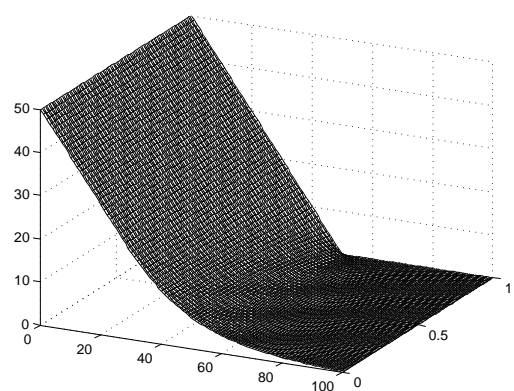

(a) $V$

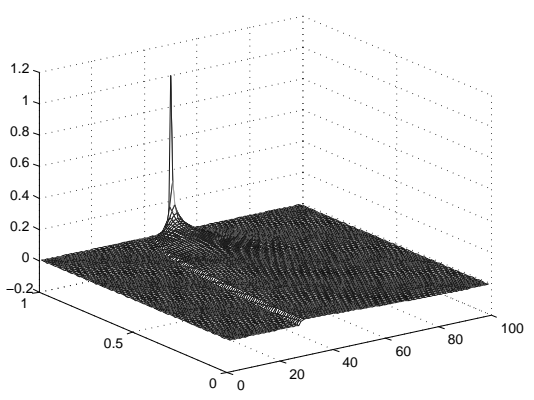

(c) $\Gamma$

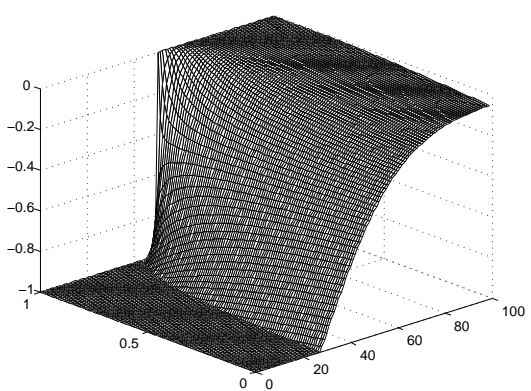

(b) $\Delta$

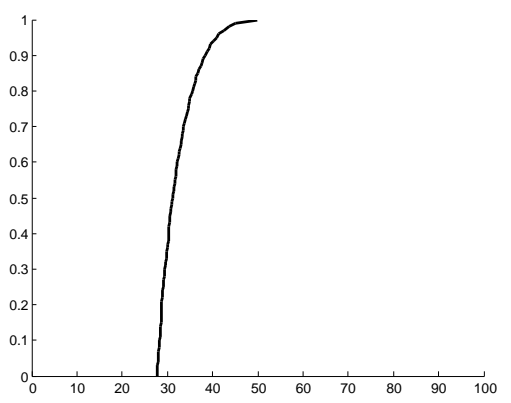

(d) Optimal exercise curve

Fig. 3 Computed value $V, \Delta, \Gamma$ and the optimal exercise curve for Test 3.

depicted in Figure 3(d). On this curve, both (2) and (3) become equalities. All of the numerical results demonstrate that our numerical method produces practically useful and meaningful solutions when applied to the American option pricing problem.

\section{Conclusions}

Motivated by conventional interior methods in constrained optimization, in this work we have proposed an interior penalty approach to the finite-dimensional large-scale LCP governing financial derivative valuation. The approach is to approximate the LCP by a nonlinear algebraic equation containing a penalty term of the form of the reciprocal of the solution. We have shown that the penalty equation has a solution in the interior of the feasible region of the original LCP. A convergence theory for the solution to the penalty equation has been established and a smooth Newton method has been proposed for solving the nonlinear penalty equation. Numerical experiments using differential LCPs in two spatial dimensions and a differential LCP in space and time for valuing American vanilla put options have been carried out. Numerical re- 
sults have demonstrated that the computed rates of convergence are consistent with theoretical one and that the method is efficient and effective for solving practical problems.

\section{Acknowledgements}

Kai Zhang wishes to thank the supports from the Philosophy and Social Science Program of Guangdong Province (Grant No. GD13YYJ01) and the MOE Project of Key Research institute of Humanities and Social Sciences at Universities (Grant No. 14JJD790041). Project 11001178 partially supported by National Natural Science Foundation of China.

\section{References}

1. L. Angermann and S. Wang, Convergence of a fitted finite volume method for the penalized Black-Scholes equation governing European and American Option pricing, Numer. Math., 106, 1-40 (2007).

2. A. Bensoussan and J.L. Lions, Applications of Variational Inequalities in Stochastic Control North-Holland, Amsterdam-New York-Oxford (1978).

3. W. Chen and S. Wang, A penalty method for a fractional order parabolic variational inequality governing American put option valuation, Comput. Math. Appl., 67, 77-90 (2014).

4. W. Chen and S. Wang, A finite difference method for pricing European and American options under a geometric Levy process, J. Ind. Manag. Optim., 11, 241-264 (2015).

5. G. Courtadon, A more accurate finite difference approximation for the valuation of options, J. Financial Economics Quant. Anal, 17, 697-703 (1982).

6. A. Damgaard, Computation of Reservation Prices of Options with Proportional Transaction Costs, Journal of Economic Dynamics and Control, 30, 415-444 (2006).

7. A. N. Daryina, A. F. Izmailov and M. V. Solodov, A class of active-set Newton methods for mixed complementarity problems, SIAM J. Optim., 36, 409-429 (2004).

8. M.H.A. Davis and T. Zariphopoulou, American Options and Transaction Fees in Mathematical Finance (eds. M.H.A. Davis et al.), Springer-Verlag (1995). Interior methods for nonlinaer optimization, SIAM Review, .44, 525-597 (2002).

9. D. Duffy, Finite Difference Methods in Financial Engineering - A Partial Differential Equation Approach, John Wiley \& Sons Ltd (2006).

10. F. Facchinei and J.S. Pang, Finite-dimensional Variational Inequalities and Complementarity Problems, Vol. I 8 II, Springer Series in Operations Research, SpringerVerlag, New York (2003).

11. M.C. Ferris, J.S. Pang, Engineering and economic applications of complementarity problems, SIAM Rev.,39, 669-713 (1997).

12. A. Forsgren, P.E. Gill and M.H. Wright, Interior methods for nonlinear optimization, SIAM Rev.,44, 525-597 (2002).

13. R. Glowinski, Numerical Methods for Nonlinear Variational Problems Springer-Verlag, New York-Berlin-Heidelberg-Tokyo (1984).

14. A. Q. M. Khaliq, D. A. Voss and S. H. K. Kazmi, A linearly implicit predictor-corrector scheme for pricing American options using a penalty method approach, J. Banking Finance, 30, 489-502 (2006).

15. C.C. Huang and S. Wang, A Power Penalty Approach to a Nonlinear complementarity problem, Operations Research Letters, 38, 72-76 (2010).

16. C.C. Huang and S. Wang, A penalty method for a mixed nonlinear complementarity problem, Nonlinear Analysis, 75, 588-597 (2012).

17. C. Kanzow, Global optimization techniques for mixed complementarity problems, $J$. Glob. Optim., 16, 1-21 (2000). 
18. D. Kinderlehrer and G. Stampacchia, An introduction to variational inequalities and their applications, Academic Press, New York (1980).

19. D.C. Lesmana and S. Wang, An upwind finite difference method for a nonlinear BlackScholes equation governing European option valuation under transaction costs, Applied Mathematics \& Computation, 219, 8811-8828 (2013).

20. W. Li and S. Wang, Pricing American options under proportional transaction costs using a penalty approach and a finite difference scheme, J. Ind. Manag. Optim., 9, 365-398 (2013).

21. W. Li and S. Wang, A numerical method for pricing European options with proportional transaction costs, J. Glob. Optim., 60, 59-78 (2014).

22. D. Li and M. Fukushima, Smoothing Newton and Quasi-Newton Methods for Mixed Complementarity Problems, Comput. Optim. Appl., 17, 203-230 (2000).

23. B.F. Nielsen, O. Skavhaug and A. Tveito. Penalty and front-fixing methods for the numerical solution of American option problems. J. Comp. Fin., 5, 69-97 (2001).

24. J.M. Ortega, W.C. Rheinboldt, Iterative solution of nonlinear equations in several variables, Academic Press (1970)

25. F.A. Potra and Y. Ye, Interior-point methods for nonlinear complementarity problems. J. Optim. Theory App., 88, 617-642 (1996).

26. R. Seydel, Tools for Computational Finance (5th Ed.), Springer Verlag, London (2012)

27. R. S. Varga. Matrix Iterative Analysis. Prentice-Hall, Engelwood Cliffs, NJ (1962).

28. S. Wang. A novel fitted finite volume method for the Black-Scholes equation governing option pricing. IMA J. Numer. Anal., 24, 699-720 (2004).

29. S. Wang, A power penalty method for a finite-dimensional obstacle problem with derivative constraints, Optim. Lett., 8, 1799-1811 (2014).

30. S. Wang, A penalty approach to a discretized double obstacle problem with derivative constraints, J. Glob. Optim., 62, 775-790 (2015).

31. S. Wang and X.Q. Yang, A power penalty method for linear complementarity problems, Operations Research Letters, 36, 211-214 (2008).

32. S. Wang and X.Q. Yang, A power penalty method for a bounded nonlinear complementarity problem, Optimization, 64, 2377-2394 (2015).

33. S. Wang, X.Q. Yang and K.L. Teo, Power penalty method for a linear complementarity problem arising from American option valuation, J. Optim. Theory App., 129, 227-254 (2006).

34. S. Wang, S. Zhang and Z. Fang, A superconvergent fitted finite volume method for Black-Scholes equations governing European and American option valuation, Numerical Partial Differential Equations, 31, 1190-1208 (2015).

35. P. Wilmott, J. Dewynne and S. Howison, Option Pricing: Mathematical models and computation, Oxford Financial Press, Oxford (1993).

36. N. Yamashita anf M. Fukushima, M., On stationary points of the implicit Lagrangian for nonlinear complementarity problems, Journal of Optimization Theory and Applications, 84, 653-663 (1995).

37. K. Zhang and S. Wang, Convergence property of an interior penalty approach to pricing American option, J. Ind. Manag. Optim., 7, 435-447 (2011).

38. K. Zhang and S. Wang, Pricing American bond options using a penalty method. Automatica, 48, 472-479 (2012). 